\title{
The influence of Transversus Abdominis Muscle Release (TAR) for complex incisional hernia repair on the intraabdominal pressure and pulmonary function
}

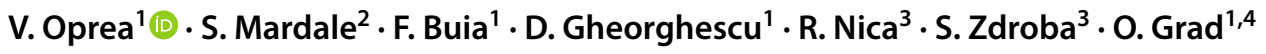

Received: 13 January 2021 / Accepted: 8 March 2021 / Published online: 22 March 2021

(C) The Author(s), under exclusive licence to Springer-Verlag France SAS, part of Springer Nature 2021

\begin{abstract}
Introduction Among many other techniques for Abdominal Wall Reconstruction (AWR), posterior component separation with Transversus Abdominis Release (TAR), continues to gain popularity and it is increasingly used with promising longterm results. Our goal was to evaluate the influence of TAR with mesh retromuscular reinforcement on the intra-abdominal pressure (IAP) and respiratory function in a series of patients with complex incisional hernias (IH).

Methods Since November 2014 through February 2019, patients with TAR were identified in the Clinical Department of Surgery database and were retrospectively reviewed. Outcome measures include: demographics, pre- and perioperative details, preoperative and postoperative IAP and plateau pressure (PP).

Results One-hundred-and-one consecutive TAR procedures (19.7\% from all incisional hernia repairs) were analyzed. Mean age was 63 years with a mean Body Mass Index (BMI) of $31.85 \mathrm{~kg} / \mathrm{m}^{2}$ (25-51). Diabetes and Chronic Obstructive Pulmonary Disease (COPD) were the main major comorbidities. Mean hernia defect area was $247 \mathrm{~cm}^{2}\left(104-528 \mathrm{~cm}^{2}\right)$.

Conclusion TAR is a safe and sound procedure with acceptable modifications of the IAP morbidity and recurrence rate when correctly performed on the right patient.
\end{abstract}

Keywords Complex incisional hernia $\cdot$ Posterior component separation · Transversus Abdominis Release $\cdot$ Intra-abdominal pressure $\cdot$ Abdominal perfusion pressure $\cdot$ Plateau pressure

\section{Introduction}

There is strong evidence that incisional hernias (IH) increased in their incidence despite all modern techniques, technologies and surgical "know-how" [1,2]. More than that, complex IH as defined some years ago by Slater et al., also increased their incidence [3]. This leads to several

V. Oprea

opreacv31@gmail.com

1 Clinical Department of Surgery, "Constantin Papilian" Emergency Military Clinical Hospital, No. 22 General Traian Mosoiu Street, Cluj-Napoca, Cluj County, Romania

2 Department of Radiology, "Constantin Papilian" Emergency Military Clinical Hospital, Cluj-Napoca, Romania

3 Intensive Care Unit, "Constantin Papilian" Emergency Military Clinical Hospital, Cluj-Napoca, Romania

4 2nd Department of Surgery, Iuliu Hatieganu" University of Medicine and Pharmacy, Cluj-Napoca, Romania serious challenges: a technical one, because in such patients, it is difficult to achieve the goals of the abdominal wall reconstruction (AWR), and a post-operative one, due to increased wound morbidity (surgical-site occurrences and surgical-site infections), increased intra-abdominal hypertension, respiratory failure, abdominal compartment syndrome and even mortality [4].

There is also strong evidence that mesh repair is compulsory and also that the sub-lay mesh positioning is the golden standard [5,6]. According to this evidence, the "golden standard" Rives-Stoppa repair is not always a valid option in complex IH due to its limited anatomical and physiological resources for reconstructing the abdominal wall with adequate tension. The recently developed posterior component separation with Transversus Abdominis Muscle Release (TAR) seems to fulfill the goals of the AWR. By its lateral extension towards the semilunar line, TAR ensures a large space in the pre-peritoneal retro-muscular plane for a large, ordinary polypropylene mesh. Due to preservation of the neuro-vascular bundles of the rectus muscle, release of the 
"hoop" tension, and closure of the anterior rectus fascia in more than $95-98 \%$ of the patients, TAR re-establishes an anatomical and physiological sound abdominal wall [7].

There are few studies on large series of complex incisional hernias so, our goal was to evaluate the impact of the anatomy of the abdominal wall defect and of the abdominal wall reconstruction via TAR on the post-operative abdominal wall physiology in terms of intra-abdominal pressure (IAP) and pulmonary pressure expressed as plateau pressure (PP).

\section{Materials and methods}

\section{Patients}

After obtaining Institutional Ethics Committee approval, a retrospective review was conducted to analyze relevant variables, which included patient demographics, hernia characteristics, operative details, and postoperative outcomes. The main outcomes of interest were intra-abdominal pressure and plateau pressure. All files of the IH patients admitted between November 2014 and February 2019, were retrospectively analyzed. Complex IH was defined according to Slater et al. criteria [3]. Large IH was defined as a ventral IH with a fascial defect $\geq 10 \mathrm{~cm}$ width with or without mesh recurrence; lateral incisional hernias were also included. Patients with class IV Ventral Hernia Working Group (VHWG) classification [8] and emergent presentations were excluded. Patients' written informed consent was obtained before surgery.

\section{CT protocol investigation}

All patients were preoperatively evaluated with a native abdomino-pelvic CT scan. All pre-operative CT scans were reviewed by the surgeon and a radiologist. After the crossreview, all inconsistencies of the measurements were eliminated. The length and the width of the defects were identified in their maximal dimensions and carefully measured. In most of the patients, the defect was equated with an ellipse and its surface was calculated using the formula $1 / 2$ length $\times 1 / 2$ width $\times \pi$. The values were approximated to the nearest hundred or thousand. When multiple associated defects were detected in various position and different dimensions (upper right quadrant and medial), the larger was considered. The width of the both rectus sheath (RW) has been also measured. The ratio of their sum to the width of the defect (DW) was the basis for TAR indication (Carbonell's equation) [9]. The volume of the abdominal cavity (ACV) and of the incisional hernia sac (IHV) was calculated according to Tanaka method [10]. The peritoneal cavity volume (PV) was obtained by adding up ACV and IHV. The peritoneal index was calculated as the ratio between IHV and PV. All the values were manually collected because there was no computer software; differences larger than $\pm 10 \%$ between surgeon's and radiologist's measurements were reassessed until an agreement was reached.

\section{Preoperative optimization}

Patient optimization included smoking and tobacco use cessation 1 month prior to elective surgery, glycemic control ensuring an $\mathrm{HbA} 1 \mathrm{c}$ level less than 7\% and improvement of cardiovascular and ventilatory capacity. Nutritional status was corrected until adequate level of total proteins, albumins and ions were achieved.

\section{Functional evaluation}

Respiratory function was evaluated by computerized spirometry (vital capacity - VC in $\mathrm{ml}$, forced expiratory volumeFEV1 in ml, and the ratio between them-FEV1/VC) before surgery and 1 month after abdominal wall reconstruction so as to avoid errors and bias induced by incomplete deep breathing as a consequence of an unhealed abdomen. Normal values or predicted values were calculated according to age, sex and height. When $\mathrm{FEV}_{1}$ was below $88 \%$ from the predicted value, an obstructive pulmonary disease was considered and it was stratified as follows: mild-when FEV ${ }_{1}$ is $80 \%$ of the predicted value; moderate $-\mathrm{FEV}_{1}$ ranges between 50 and $79 \%$ of the predicted value; and severewith $\mathrm{FEV}_{1}$ between 30 and $49 \%$ of the predicted value. Normal value of FEV1/VC was considered around $75 \%$ or 0.75 . When VC was decreased but the ratio was normal, a restrictive lung insufficiency was considered and was attributed to the abdominal wall defect among other pathologies.

\section{Intra-abdominal pressure}

The standard method for indirectly measuring IAP is to measure the intra-vesicular pressure [11]. Briefly, the bladder drainage system was clamped just distal to the connection of the urinary catheter to the drainage bag. An 18-gauge needle was then inserted into the sampling port and connected via a sterile tube to the pressure transducer using two three-way stopcocks. A standard infusion bag of normal saline was attached to one stopcock, and a 60-ml syringe was connected to the second stopcock. Sterile saline $(50-100 \mathrm{ml})$ was injected into the bladder. Measurements were taken at end-expiration while patients were in complete supine position and with the transducer zeroed at the symphysis pubis level. Intra-abdominal pressure was recorded before surgery, immediate after the end of the procedure, 24 , and $48 \mathrm{~h}$ after TAR on the awakened patient. Intra-abdominal hypertension (IAH) is defined by a sustained or repeated elevation 
in IAP $\geq 12 \mathrm{mmHg}$. IAH was graded as follows: Grade I, IAP $=12-15 \mathrm{mmHg}$; Grade II, IAP $=16-20 \mathrm{mmHg}$; Grade III, IAP $=21-25 \mathrm{mmHg}$; and Grade IV, IAP $>25 \mathrm{mmHg}$ [12]. Likewise, abdominal compartment syndrome (ACS) was defined as IAP $>20 \mathrm{mmHg}$ in combination with at least one organ failure. In addition, abdominal perfusion pressure (APP) was calculated by subtracting IAP from mean arterial pressure (MAP) measurements. Mean APP $<60 \mathrm{mmHg}$ was considered abnormal.

Plateau pressure $\left(P_{P L A T}\right)$ is the pressure applied to small airways and alveoli during positive-pressure mechanical ventilation. It is measured during an inspiratory pause on the mechanical ventilator and it was recorded after $20 \mathrm{~min}$ of the onset of the procedure and 20 min after the anterior fascia was closed. The difference between first and last record of the plateau pressure was noted as $\Delta$ plateau pressure. When the difference exceeds $6 \mathrm{mmHg}$, the patient was kept intubated for $24 \mathrm{~h}$.

\section{Procedure}

The indication for TAR was established preoperatively using the formula $2 \mathrm{RW}: \mathrm{DW}=2: 1$. All the procedures were performed under general anesthesia according to the technique described by Novitsky $[13,14]$. For the posterior fascia closure, a slowly resorbable suture (2-0 polydyoxanone) was used as a running suture. Mesh was fixed with transfascial slowly absorbable suture or with cyanoacrylate (Histoacryl ${ }^{\circledR}$ B Braun). Anterior fascia was closed with a running slowly absorbable suture (1 polydyoxanone). When repairing a mesh recurrence, the mesh was completely removed whenever possible. Monofilament macroporous medium-weight polypropylene mesh was used for all circumstances when the anterior fascia was completely closed. When the linea alba could not be restored, a heavy weight polypropylene mesh was used.

\section{Follow-up}

Patients were followed up 1 month, 6 months and yearly after TAR by direct examination if possible. For the goals of this paper, only 1 month follow-up was considered.

\section{Statistical analysis}

Data were tabulated as mean \pm standard deviation (sd). Continuous variables were analyzed by ANOVA variance test followed by unpaired two tails Student's $t$ test assuming unequal variance and the binary outcomes with the $\chi^{2}$ test. Pearson correlation $(r)$ was used with the regression equation. Multivariate logistic regression models were built with PI, IAP as the outcomes of interest for major determinants of severe postoperative complications, adjusting for identified confounders. In addition to the variables of interest, the following were included for adjustment: age, rank of recurrence, severity of comorbidities score, body mass index (BMI), length, width and area of the defect, ACV, IHV, PV, and postoperative intra-abdominal pressure. Multivariate models were calculated with the linear logistic regression and the results were shown with the Odds ratio (OR) and the $95 \%$ confidence interval (CI). Probabilities smaller than 0.05 were considered as statistically significant. SPSS statistic version 23.0, 2018 (Chi, Ill) was used to perform the statistical analysis.

\section{Results}

All data had a normal distribution (one-sample Kolmogorov-Smirnov and one-sample Chi-square tests). In the referral interval, 306 patients with $\mathrm{IH}$ were operated in the Department of Surgery. Out of these, 101 (33\%) meet the inclusion criteria and were operated by TAR. Demographic data and preoperative hernia characteristics are represented in Table 1.

Table 2 outlines the CT scan characteristics for all $\mathrm{IH}$.

Mean preoperative VC was $2220.94 \pm 317.24$ (1675-2900) $\mathrm{ml}$. All values were lower than the predicted with 3 to $40 \%$. In 31 patients, the reduction was between 3 and $17 \%$, in 22 patients from 18 and $24 \%$, in 31 from

Table 1 Demographics and preoperative hernia characteristics

\begin{tabular}{ll}
\hline Characteristics/variables & Mean \pm standard deviation (min-max) \\
\hline Number of patients/gender & $101 / 45$ males \\
Age (years) & $63.78 \pm 6.78(43-79)$ \\
Onset of symptoms (months) & $61.49 \pm 35.3(12-148)$ \\
BMI (kg/m $\left.{ }^{2}\right)$ & $31.85 \pm 4.57(25-51)$ \\
ASA & $2-87$ patients; $3-14$ patients \\
Co-morbidities (usually more & 37 patients \\
than 1) diabetes & 27 patients \\
COPD & 27 patients \\
Active smoker & 18 patients \\
Retired smokers & 51 patients \\
Cardiac & 1 patient \\
Immune suppression & Medial-90 patients \\
Hernia location & Parastomal-4 patients \\
& Lateral-7 patients \\
& RUQ-4 \\
Primary IH & RLQ-3 \\
Recurrent IH & 74 patients \\
\hline
\end{tabular}

$B M I$ body mass index, ASA American Society of Anesthesiologists Score, $C O P D$ chronic obstructive pulmonary disease, $R U Q$ right upper quadrant, $R L Q$ right lower quadrant, $I H$ incisional hernia 
Table 2 Computed tomography variables of incisional hernia anatomy

\begin{tabular}{lll}
\hline Variables & $\begin{array}{l}\text { Mean } \pm \text { standard } \\
\text { deviation }(\mathrm{sd})\end{array}$ & Minimum/maximum \\
\hline Length of the defect $(\mathrm{cm})$ & $18.24 \pm 4.11$ & $11-28$ \\
Width of the defect $(\mathrm{cm})$ & $17.18 \pm 3.29$ & $11-28$ \\
Area $\left(\mathrm{cm}^{2}\right)$ & $247.12 \pm 79.03$ & $104-528$ \\
Rectus sheath width (sum) & $7.78 \pm 1.22$ & $5-10$ \\
$\quad(\mathrm{~cm})$ & & \\
TAR index (DW: RW) & $2.25 \pm 0.47$ & $1.37-4$ \\
ACV $\left(\mathrm{cm}^{3}\right)$ & $9297.15 \pm 1452.3$ & $5017-12,138$ \\
IHV $\left(\mathrm{cm}^{3}\right)$ & $4231 \pm 800.96$ & $2017-5983$ \\
PV $(\mathrm{ACV}+\mathrm{IHV})\left(\mathrm{cm}^{3}\right)$ & $13,521 \pm 1999.07$ & $8498-18,121$ \\
PI $(\mathrm{ACV} / \mathrm{PV})$ & $0.307 \pm 0.03$ & $0.22-0.41$ \\
\hline
\end{tabular}

TAR Transversus Abdominis Release, $A C V$ abdominal cavity volume, $I H V$ incisional hernia volume, $P V$ peritoneal volume, $P I$ Peritoneal Index

25 to $34 \%$ and in 17 over $37 \%$. A strongly negative Pearson correlation was found between the length of the defect $(r=-0.103 ; p=0.305)$, width of the defect $(r=-0.321$; $p=0.001)$, and preoperative plateau pressure $(r=-0.201$; $p=0.044)$. Even if the absolute value of $r$ was not highly significant, the distribution of the plots around the regression line argued this statement (data not shown).

Preoperatively, the value of $\mathrm{FEV}_{1}$ was decreased in all patients with $5-41 \%$ than predicted value (mean $1581.92 \pm 267.09 \mathrm{ml}$; minimum $1055 \mathrm{ml}$; maximum $2340 \mathrm{ml}$ ). According to $\mathrm{FEV}_{1}$ values, 32 patients were without respiratory insufficiency (reduction of $\mathrm{FEV}_{1}$ with 9-19\%) and 69 patients with respiratory obstructive and restrictive symptoms (reduction of $\mathrm{FEV}_{1}$ with $21-41 \%$ ). Width of the defect, area, IHV and preoperative plateau pressure were negatively influenced $\mathrm{FEV}_{1}$ decrease (width $r=-0.220 ; p=0.027$, area $r=-0.209 ; p=0.036$, IHV $r=-0.205 ; p=0.04$ and plateau pressure $r=-0.247$; $p=0.013$ ).

The value of mean preoperatively $\mathrm{FEV}_{1} / \mathrm{VC}$ was slightly decreased compared to normal value $(0.71 \pm 0.1)$. In 38 patients with normal ratio, both $\mathrm{VC}$ and $\mathrm{FEV}_{1}$ were equally reduced and all these patients had large volumes of the incisional hernia sac (IHV). This was demonstrated by a significant negative correlation with the IHV $(r=-0.226$; $p=0.023)$. Extreme lower values of the ratio (0.46-0.64) were encountered in 28 patients; $38 \%$ were known with COPD and for them, the ratio was decreased due to the extreme decrease of $\mathrm{FEV}_{1}$. For the rest of them, the ratio was decreased due to the both components.

At 1 month postoperatively, VC increased to a mean of $2785.64 \pm 353.5$ (min 2000-max 3600) $\mathrm{ml}$; compared with the preoperative value, the difference was statistically significant (Student's $t$ test $-p<0.001$; ANOVA F $=3.024$,
Table 3 Intra- and post-operative characteristics of the pressure variable

\begin{tabular}{lll}
\hline Variables & $\begin{array}{l}\text { Mean } \pm \text { stand- } \\
\text { ard deviation } \\
(\mathrm{sd})\end{array}$ & $\begin{array}{l}\text { Mini- } \\
\text { mum/ } \\
\text { maximum }\end{array}$ \\
\hline Preoperative plateau pressure $(\mathrm{mmHg})$ & $14.88 \pm 3.56$ & $10-22$ \\
Postoperative plateau pressure (mm Hg) & $19.9 \pm 4.16$ & $12-30$ \\
$\Delta$ plateau (mmHg) & $5.03 \pm 1.27$ & $2-9$ \\
Postoperative IAP (mmHg) & $14.64 \pm 2.83$ & $10-20$ \\
POD 1 IAP (mmHg) & $18.17 \pm 2.84$ & $12-25$ \\
POD 2 IAP (mmHg) & $15.87 \pm 1.79$ & $11-20$ \\
\hline
\end{tabular}

TAR Transversus Abdominis Release, IAP intra-abdominal pressure, $\Delta$ plateau difference between postoperative and preoperative plateau pressure

$p<0.001) . \mathrm{FEV}_{1}$ also recorded increased values ranging from 1300 to $2800 \mathrm{ml}$ and a mean of $2092.3 \pm 335.9 \mathrm{ml}$. The difference was significant in relation with the preoperative value (Student's $t$ test $-p<0.001$; ANOVA F $=2.58$, $p<0.001)$. Related to the predicted values, the decrease was lower than the preoperative both values with $2-27 \%$. In patients with COPD, the increased of $\mathrm{FEV}_{1}$ was almost zero; comparing the means of this subgroup of patients, the difference was insignificant (Student's $t$ test $-p=0.327$; ANOVA $\mathrm{F}=1.27 ; p=0.211$ ). A significant increase of $\mathrm{FEV}_{1}$ was documented in 52 patients with restrictive respiratory insufficiency in whom this was with over $40 \%$ to preoperative values (Student's $t$ test $-p<0.001$; ANOVA $\mathrm{F}=4.19 p=0.002)$. Mean postoperative $\mathrm{FEV}_{1} / \mathrm{VC}$ ratio was $0.74 \pm 0.07$ ( $\min 0.54-\max 0.9$ ); the difference was significant compared to preoperative mean (Student's $t$ test $-p<0.001$; ANOVA F $=3.19 p=0.006)$. Postoperatively, the number of patients with normal ratio (64) was higher than the preoperative one (48) and the difference was significant (Chi-square test $\chi^{2}=23.8 ; p=0.0017$ ). There was no modification of the ratio in patients with COPD, postoperative values being almost similar with the preoperative ones.

Preoperative intra-abdominal pressure varied between 4 and $9 \mathrm{mmHg}$ with a mean of $6.15 \pm 1.56 \mathrm{mmHg}$. In a univariate analysis, preoperative IAP was strongly negative correlated with the width of the defect $(p=0.029)$, area of the defect $(p=0.019)$, IHV $(p<0.001)$, and with PI $(r=-0.803$, $r^{2}$ linear $\left.=0.6, p<0.001\right)$. When the logistic regression was applied, the only valid correlation of the preoperative IAP was with the PI $(\mathrm{OR}=1.82,95 \%$ CI $1.04-3.18, p=0.032)$.

Intra-operative findings are detailed in Table 3 . There was no difference between preoperative and intra-operative values of the defect (data not shown).

In 11 patients with a TAR index between 0.32 and 0.4 , the posterior fascia could not be closed, so a peritoneal flap in five patients and an omental patch in the rest of them 
was used to separate the mesh from the abdominal content. No biologic or absorbable meshes were available. Anterior fascia was completely closed in 94 patients (93\%) and for the rest of them, a bridged repair with mesh fixation to the edges of the defect was performed.

Immediate postoperative IAP ranged between 10 and $20 \mathrm{mmHg}$ with a mean value of $14.64 \mathrm{mmHg}$ and it was significantly higher compared to the preoperative value (Student's $t$ test $-p<0.001)$. All patients developed intraabdominal hypertension in varying degrees: grade $I$ (12-15 $\mathrm{mmHg})-63$ patients, and grade II (16-20 $\mathrm{mmHg})-$ 38 patients immediately after the end of the procedure.

In the first postoperative day (POD 1), IAP increased to a mean value of $18.17 \mathrm{mmHg}$ (statistically significant compared to the mean value recorded immediately postoperatively; $p=0.017$ Student's $t$ test). The distribution of patients according with the grade of IAH was also changed: grade $I-22$ patients, grade $I I-42$ patients and grade $I I I-37$ patients. No organ dysfunction was recorded, urinary output being in normal range for all patients. Values of the IAP for the second postoperative day (POD2) and of the urinary out are represented in Fig. 1.

In Table 4 are listed the main variables correlated with the postoperative IAP in the univariate analysis. Because all data were outside confidence interval of the linear regression equation, even if the statistical significance was high, a binary logistic regression was performed. The only variables correlated with the postoperative IAP were IHV, PI, RSW, and preoperative IAP. Data are shown in Table 4.

The mean value for the postoperative plateau pressure was significantly higher than the mean value before abdominal wall reconstruction $(p<0.01)$. For 17 patients, the difference between postoperative and preoperative value of plateau pressure ( $\Delta$ plateau) was over 6 and they were kept intubated for $24 \mathrm{~h}$. Before extubation, the value of plateau pressure was almost similar with preoperative values (data not shown). The same factors that control IAP are also determinants for plateau pressure in the univariate
Fig. 1 Distribution of patients in relation with the level of intra-abdominal hypertension (IAH). Grade I, IAP $=12-15 \mathrm{mmHg}$; Grade II, $\mathrm{IAP}=16-20 \mathrm{mmHg}$; Grade III, IAP $=21-25 \mathrm{mmHg}$; IPO immediate after the end of the procedure, $P O D 1$ postoperative day $1, P O D 2$ postoperative day 2

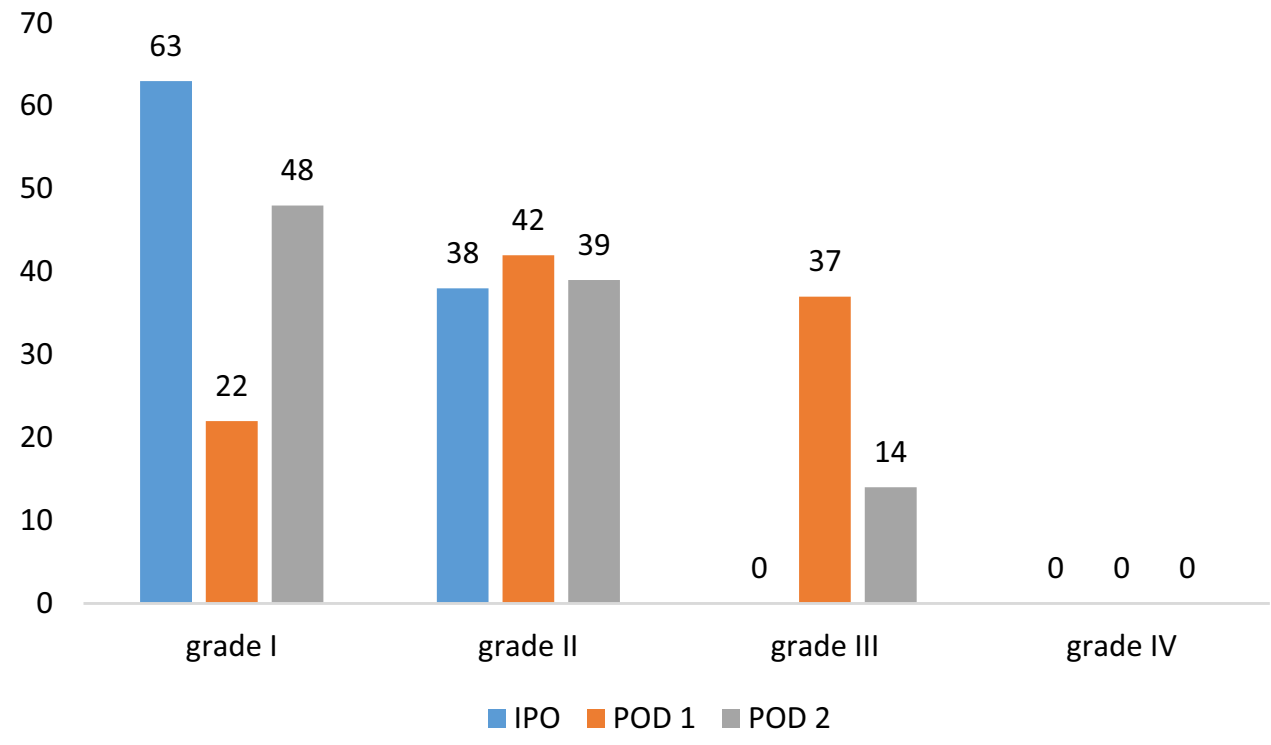

Table 4 Significant factors influencing postoperative intra-abdominal pressure (IAP) (univariate analysis and logistic regression)

\begin{tabular}{lll}
\hline Variables & $\begin{array}{l}\text { Postoperative IAP (univari- } \\
\text { ate analysis) }\end{array}$ & Postoperative IAP (logistic regression) \\
\hline Length of the defect & $r=0.356 ; p<0.001$ & OR $0.2 ; 95 \% \mathrm{CI}=0.1-0.5 ; p=0.8$ \\
Width of the defect & $r=0.242 ; p=0.015$ & OR $0.4 ; 95 \% \mathrm{CI}=0.1-0.7 ; p=0.08$ \\
Area of the defect & $r=0.395 ; p<0.001$ & OR $0.4 ; 95 \% \mathrm{CI}=0.2-0.7 ; p=0.6$ \\
ACV & $r=-0.155 ; p=0.122$ & OR $0.2 ; 95 \% \mathrm{CI}=0.1-0.5 ; p=0.8$ \\
IHV & $r=0.468 ; p<0.001$ & OR $1.12 ; 95 \% \mathrm{CI}=0.91-2.13 ; p=0.002$ \\
PI & $r=0.710 ; p<0.001$ & OR $0.78 ; 95 \% \mathrm{CI}=0.45-1.37 ; p=0.011$ \\
Preoperative IAP & $r=-0.634 ; p<0.01$ & OR $0.85 ; 95 \% \mathrm{CI}=0.48-1.49 ; p=0.029$ \\
Rectus sheath width (sum) & $r=0.096 ; p=0.341$ & OR $1.09 ; 95 \% \mathrm{CI}=0.61-1.93 ; p=0.014$
\end{tabular}

$A C V$ abdominal cavity volume, $I H V$ incisional hernia volume, $P I$ Peritoneal Index, $O R$ odd ratio, $95 \% C I$ confidence interval 
Table 5 Significant factors influencing postoperative plateau pressure (univariate analysis and logistic regression)

\begin{tabular}{lll}
\hline Variables & $\begin{array}{l}\text { Postoperative plateau pressure (univariate } \\
\text { analysis) }\end{array}$ & Postoperative plateau pressure (logistic regression) \\
\hline Length of the defect & $r=0.197, r^{2}=0.039, p=0.049$ & OR $0.2 ; 95 \% \mathrm{CI}=0.1-0.5 ; p=0.8$ \\
Width of the defect & $r=0.213, r^{2}=0.043, p=0.041$ & OR $0.6 ; 95 \% \mathrm{CI}=0.1-0.9 ; p=0.3$ \\
Area of the defect & $r=0.220, r^{2}=0.048, p=0.027$ & OR $0.2 ; 95 \% \mathrm{CI}=0.1-0.5 ; p=0.8$ \\
ACV & $r=-0.201, r^{2}=0.04, p=0.044$ & OR $0.2 ; 95 \% \mathrm{CI}=0.57-3.06 ; p=0.007$ \\
IHV & $r=0.301, r^{2}=0.091, p=0.002$ & OR $1.19 ; 95 \% \mathrm{CI}=0.52-2.73 ; p=0.019$ \\
PI & $r=0.551, r^{2}=0.350, p=0.001$ & OR $1.64 ; 95 \% \mathrm{CI}=0.0 .68-3.99 ; p=0.001$ \\
Preoperative IAP & $r=-0.608, r^{2}=0.369, p=0.001$ & OR $0.2 ; 95 \% \mathrm{CI}=0.1-0.5 ; p=0.8$ \\
Postoperative IAP & $r=0.658, r^{2}=0.433, p=0.001$ & OR $1.09 ; 95 \% \mathrm{CI}=0.48-2.45 ; p=0.022$ \\
Rectus sheath width (sum) & $r=0.21,7 r^{2}=0.044, p=0.041$ & OR $0.4 ; 95 \% \mathrm{CI}=0.1-08 ; p=0.07$
\end{tabular}

$A C V$ abdominal cavity volume, $I H V$ incisional hernia volume, $P I$ Peritoneal Index, $O R$ odd ratio, 95\% CI confidence interval

analysis but after binary logistic regression only, ACV, IHV, PI and postoperative IAP remain as significant (Table 5).

The mean value for APP in POD 1 was $71.99 \pm 10.09$ (60-93) mmHg. 17 patients with PI higher than 0.3, with grade III IAH, and $\Delta$ plateau $>6 \mathrm{mmHg}$, were kept intubated for the next $24 \mathrm{~h}$, the mean value of APP was $60.2 \pm 1.7 \mathrm{mmHg}$ and significantly lower than for the rest of the patients $(71.44 \pm 9.6 \mathrm{mmHg} ; p<0.001$; Student's $t$ test). In POD 2, the mean value of APP increased to $78.2 \pm 9.39 \mathrm{mmHg}$; for the intubated patients, the mean value of the APP increased to a mean of $71.8 \pm 8.6 \mathrm{mmHg}$ and was slightly lower than of the other patients $(74.6 \pm 6.34$; $p=0.08)$. A negative strong correlation with the PI was recorded for APP ( $r$ Pearson $=-0.873 ; p=0.004)$ in a univariate analysis. When logistic regression was applied, ACV (OR 1.19; 95\% CI =0.52-2.67; $p=0.027$ ), IHV (OR $1.29 ; 95 \% \mathrm{CI}=0.72-2.87 ; p=0.018)$, PI (OR 2.83; $95 \%$ $\mathrm{CI}=1.52-3.84 ; p=0.015)$, postoperative plateau pressure (OR 2.19; 95\% CI $=1.52-3.67 ; p=0.017$ ) and BMI (OR $0.68 ; 95 \% \mathrm{CI}=0.67-2.67 ; p=0.021)$ were the factors which significantly influence the values of the APP.

No mortality was recorded at 30 days. Systemic morbidity was noted in 38 patients and consisted of: urinary tract infection (two patients), prolonged paralytic ileus -5 to 8 days (11 patients), deep vein thrombosis (one patient), deep vein thrombosis with limited pulmonary embolism (one patient), pneumonia (two patients), and prolonged simple $\mathrm{O}_{2}$ administration for 21 patients. All pulmonary events occurred in patients with pre-existing lung damage. Paralytic ileus was more frequent in diabetic patients (nine of eleven). Female gender was more frequently exposed to develop systemic morbidity (71 vs 29\%). Wound complications were recorded in $11(29 \%)$ of the patients with systemic postoperative morbidity. These surgical-site occurrences (SSOs) were hematomas in three patients, seromas in two patients and surgicalsite infections (SSIs) in the rest of them. Re-intervention was necessary for hematoma patients with an un-eventful recovery and for the half of the patients with SSIs. No mesh debridement was necessary. In all cases of SSIs, the wound was healed by active therapy with negative pressure between 8 and 21 days. Hospital stay ranged between 5 and 38 days (mean of $7.21 \pm 2.8$ days).

Follow-up varied between 12 and 51 months with mean of 32 months. At 12 months, 91 patients were directly examined and seven recurrences were recorded. Most of them (five patients) were recorded in the first 35 patients between 5 and 11 months after surgery. After the 35th repair, no early recurrence was encountered. There was no established relation between post-operative IAP, respiratory status and recurrence rate. Defect area $(\mathrm{OR}=2.81$; 95\% CI 1.69-3.72; $p=0.004)$, active smoking $(\mathrm{OR}=2.21 ; 95 \%$ CI $1.35-3.45$; $p=0.001)$, and diabetes (OR $=1.93 ; 95 \%$ CI $1.19-2.83$; $p=0.022$ ) were the only factors independently associated with recurrence rate.

\section{Discussion}

From a mechanical point of view, the $\mathrm{IH}$ is an opening in the abdominal wall layers which leads to impossibility of the wall to withstand IAP. An increased number of IH are complicated cases, which include large defects or even loss of domain, lateral incisional hernias, recurrence after mesh repair and /or chronic mesh infection. Surgery of complex IH is a difficult and thorny issue for both surgeon and patient. The main challenge for this surgery is to find the best technique for a complex given equation (patient, hernia, anatomy) which must provide a complete reconstruction of the linea alba augmented by a large and adequately placed prosthetic mesh, with minimal early and late morbidity. The field of herniology offers a large amount of data regarding surgical approach and mesh types but heterogeneity and biases limit standardization [15]. 
Few options are available but according to the principles and the goals of AWR, they restrict only to component separation techniques. Posterior component separation viaTAR is a relatively new technique which ensures the lateral extension of the retro-rectus space for a wide mesh overlap, release "hoop-tension", allows closure of the anterior fascia in most patients and preserves the AW function by preserving the neurovascular bundles for the rectus abdominis muscle.

According to Majumder et al., on a cadaveric study, after a complete TAR with large retro-muscular dissection, an average advancement of $10 \mathrm{~cm}$ for the anterior fascia and $11 \mathrm{~cm}$ for the posterior fascia on each side was obtained [16]. This can provide a significant increase of the abdominal cavity inner diameters and, subsequently, an increase of the whole visceral sac volume, without or with minimal consequences upon normal physiology. Indeed, this study reconfirms our previous findings [17]; IAP and postoperative plateau pressure maintained permissive values without altering respiratory function for 90 patients. Peritoneal index and rectus sheath's width are the only factors which influence the negative respiratory outcomes. Myofascial release of the transversus abdominis muscle can generate elevated IAP immediately following repair, clearly demonstrated by our results. All our patients developed intra-abdominal hypertension to varying degrees but no values larger then $15 \mathrm{mmHg}$ were recorded in patients with PI smaller than 0.3. Analyzing the generalized Log Odds (Fig. 2), one can observe that the main factors for unfavorable prognosis impact the outcomes of IAP from the values over 0.33 of the peritoneal index and are more expressed over the value of 0.37. A total of 37 patients $(36.62 \%)$ developed increased values of the postoperative IAP and plateau pressure directly related to an increased PI. In nine of them, the values were extreme but without developing abdominal compartment syndrome.

These elevated IAP could be considered permissible due to the fact that no organ dysfunction, except for some reversible decrease of the urinary output, as recorded. Probably, maintaining APP above the critical value of $60 \mathrm{mmHg}$ was an important contributing factor to the positive outcomes of the patients (data not shown). Under these circumstances, one could suggest that intra-abdominal hypertension or abdominal compartment syndrome immediately after an elective abdominal wall reconstruction creates a clinical aspect that must be distinguished from the settings in which they were originally described [18].

One factor that can be involved in the favorable outcomes of our patients is the abdominal compliance $\left(C_{\mathrm{ab}}\right)$. It plays a very important role in understanding the deleterious effects of un-adapted intra-abdominal volumes on IAP and organ dysfunction. Unfortunately, measurements of $C_{\mathrm{ab}}$ are difficult at the bedside. Some indirect measures are available
Fig. 2 Main factors correlated with unfavorable prognosis in relation with peritoneal index

\section{Generalized Log Odds}

\section{Coefficients $^{\mathrm{b}, \mathrm{c}}$}

\begin{tabular}{|l|c|r|r|r|r|r|}
\hline pindex & \multicolumn{1}{|c|}{ IHV $^{\mathrm{a}}$} & IAPpreop $^{\mathrm{a}}$ & IAPpostop $^{\mathrm{a}}$ & \multicolumn{1}{|c|}{ IAPD1 } & platpreop $^{\mathrm{a}}$ & platpostop $^{\mathrm{a}}$ \\
\hline .22 & 2893.00 & 7.00 & 11.00 & 15.00 & 15.00 & 20.00 \\
.23 & 2474.33 & 8.00 & 12.67 & 16.33 & 12.00 & 17.33 \\
.24 & 3054.83 & 7.67 & 11.50 & 14.83 & 11.33 & 16.00 \\
.27 & 3869.60 & 7.80 & 12.50 & 16.20 & 12.20 & 17.50 \\
.28 & 4102.67 & 8.00 & 12.44 & 16.22 & 13.22 & 18.44 \\
.29 & 3884.25 & 7.50 & 11.50 & 15.00 & 12.00 & 16.75 \\
.30 & 3954.45 & 6.82 & 14.45 & 18.36 & 14.36 & 18.55 \\
.31 & 4030.50 & 6.00 & 14.71 & 18.36 & 14.57 & 19.29 \\
.32 & 4931.40 & 5.60 & 15.20 & 19.60 & 13.60 & 19.00 \\
.33 & 4971.08 & 5.42 & 14.92 & 18.33 & 15.83 & 20.25 \\
.34 & 4793.85 & 4.46 & 17.15 & 20.46 & 18.15 & 23.69 \\
.35 & 4998.38 & 4.38 & 17.25 & 20.63 & 17.88 & 23.38 \\
.37 & 4933.00 & 4.00 & 19.00 & 22.00 & 20.00 & 28.00 \\
.38 & 3764.00 & 4.00 & 19.00 & 22.00 & 12.00 & 17.00 \\
.39 & 4917.00 & 4.00 & 19.00 & 21.00 & 21.00 & 27.00 \\
.40 & 3902.00 & 4.00 & 20.00 & 21.00 & 22.00 & 30.00 \\
.41 & 3481.00 & 4.00 & 20.00 & 21.00 & 21.00 & 30.00 \\
\hline
\end{tabular}


in mechanically ventilated patients: the $\triangle \mathrm{IAP}(=\mathrm{IAP}$ at the end of inspiration minus the IAP at the end of expiration) and the abdominal pressure variation $(\mathrm{APV}=\Delta \mathrm{IAP}$ divided by mean IAP) are such parameters, and they are inversely correlated with $C_{\mathrm{ab}}$, i.e., the higher $\triangle \mathrm{IAP}$ or APV, the lower $C_{\mathrm{ab}}$ [12]. Therefore, if we could identify patients with a low $C_{\mathrm{ab}}$, we could anticipate and select the most appropriate surgical treatment to avoid possible complications related to IAH/ACS

Preoperative evaluation of the lung function with the aid of spirometry is an easy way to differentiate if the respiratory insufficiency is obstructive or restrictive at origin. Obstructive $\mathrm{FEV}_{1}$ is reduced, so the ratio will be low. In large $\mathrm{IH}$, this can be no longer the truth due to the fact that the obstruction can be masked by the broken associated abdominal component of the respiration. When the ratio is normal or even increased due to a decrease in magnitude of $\mathrm{VC}$ and $\mathrm{FEV}_{1}$ decrease, the respiratory failure is restrictive as a consequence of the large defect and increased IHV. The surgical procedure itself improves significantly the restrictive respiratory failure by correcting the functionality of the abdominal wall.

Some limitations of the study can be considered. This is a retrospective one even if our database is prospective from a single surgical center; the number of patients is not too high but represents a quarter from all patients with incisional hernias admitted in our Department. Also, the population and hernia characteristics are not homogenous and the results can be biased by the learning curve of a new procedure. Most of the procedures ( 92 patients) were performed by a single surgeon, so the results cannot be broadly reproducible.

\section{Conclusion}

Posterior component separation with TAR is a strongly effective procedure for a stable repair of large and very large defects of the abdominal wall irrespective of their location. TAR is also effective in recreating abdominal wall volumes without severe disturbing of the IAP, plateau pressure and respiratory function when peritoneal index is lower than 0.33. Last but not least, TAR improves restrictive respiratory insufficiency through correction of the abdominal wall functionality. A correct preoperative evaluation (CT scan, respiratory, cardiovascular and metabolic) is the key for minimizing severe complications and achieving superior outcomes. Proper training and profound understanding of the abdominal wall anatomy are the cornerstones for the success.

Authors' contribution VO: study conception and design, acquisition, analysis and interpretation of data, drafting the work and revising, final approval of the version to be published; SM: acquisition, analysis and interpretation of imagistic data, final approval of the version to be published; FB, DG, RN, ZS: final approval of the version to be published; OG: interpretation of data, drafting the paper, final approval.

Funding No financial support was necessary for this study.

\section{Declarations}

Conflict of interest All authors declare no conflict of interest.

Ethical approval All procedures performed in this study involving Human participants were in accordance with the ethical standards of the institutional and/or national research committee and with the 1964 Helsinki declaration and its later amendments or comparable ethical standards.

Human and animal rights No animal was used for this study.

Informed consent Informed written consent was obtained from all patients before surgery.

\section{References}

1. Dietz UA, Winkler MS, Härtel RW, Fleischhacker A, Wiegering A et al (2014) Importance of recurrence ratting, morphology, hernia gap size, and risk factors in ventral and incisional hernia classification. Hernia 18:19-30

2. Bower C, Roth SJ (2013) Economics of abdominal wall reconstruction. Surg Clin N Am 93:1241-1312

3. Slater NJ, Montogomery AK, Berrevoet T, Carbonell AM, Chang A et al (2014) Criteria for definition of a complex abdominal wall hernia. Hernia 18:7-17. https://doi.org/10.1007/ s10029-013-1168-6

4. Mudge M, Hughes LE (1985) Incisional hernia: a 10 year prospective study of incidence and attitudes. Br J Surg 72(1):70-71

5. Holihan JL, Halawady ZM, Harris JW, Harvin J, Shah SK et al (2016) Ventral hernia: patient selection, treatment and management. Curr Probl Surg 53:307-354

6. Holihan JL, Nguyen DH, Nguyen MT, Mo J, Kao L, Liang MK (2015) Mesh location in open ventral hernia repair: a systematic review and network meta-analysis. World J Surg. https://doi.org/ 10.1007/s00268-015-3252-9 (Published on line 30 September)

7. Novitsky YW, Elliot HL, Orenstein SB, Rosen MJ (2012) Transversus abdominis muscle release: a novel approach to posterior component separation during complex abdominal reconstruction. Am J Surg 204:709-716

8. Ventral Hernia Working Group et al (2010) Incisional ventral hernias: a review of the literature and recommendations regarding the grading and technique of repair. Surgery 148:544-548

9. Carbonell A (2018) The 9th Annual Abdominal Wall Reconstruction Summit, Montana USA

10. Tanaka EY, Yoo JH, Rodrigues AJ Jr, Utyiama EM, Birollini D et al (2010) A computerized tomography scan method for calculating the hernia sac and abdominal cavity volume in complex large incisional hernias with loss of domain. Hernia 14:63-69

11. Ivatury RR (2018) Open abdomen: historical notes. In: Coccolini F, Ivaturi R, Sugrue M, Ansaloni L (eds) Open abdomen. A comprehensive practical manual. Springer International Publishing, pp 1-26 
12. Fugazzola P, Montori G, Rizoli S, Ansaloni L, Rezende-Neto J (2018) Basic research in open abdomen. In: Coccolini F, Ivaturi R, Sugrue M, Ansaloni L (eds) Open abdomen. A comprehensive practical manual. Springer International Publishing, pp 27-53

13. Gibreel W, Saar MG, Rosen MJ, Novitsky YW (2016) Technical considerations in performing posterior component separation with transverse abdominis muscle release. Hernia 20:449-459

14. Pauli EM, Rosen MJ (2013) Open ventral hernia repair with posterior component separation. Surg Clin North Am 93:1111-1113

15. Blatnick JA, Brunt ML (2019) Controversies and techniques in the repair of abdominal wall hernias. J Gastrointest Surg 23:837-845. https://doi.org/10.1007/s11605-018-3989-1

16. Majumder A, Miller HG, del Campo LM, Soltanian H, Novitsky YW (2018) Assessment of myofascial medialization following posterior component separation via transversus abdominis release in a cadaveric model. Hernia. https://doi.org/10.1007/s10029-0181771-7 (published on line 30 April)
17. Oprea VC, Rosian M, Mardale S, Grad O (2020) Is transversus abdominis muscle release sustainable for the reconstruction of peritoneal volumes? A retrospective computed tomography study. Int J Abdom Wall Hernia Surg 3:25-33

18. Kirkpatrick AW, Roberts DJ, De Waele J, Blaser AR, Malbrain MLNG, Bjorck M, Balogh ZJ, Executive Committee of the WSACS-Abdominal Compartment Society (2016) Permissive intraabdominal hypertension following complex abdominal wall reconstruction. Plast Reconstr Surg 137(4):762e-764e. https://doi. org/10.1097/PRS.0000000000001998

Publisher's Note Springer Nature remains neutral with regard to jurisdictional claims in published maps and institutional affiliations. 